\title{
Variety of Iron Oxide Inclusions in Sapphire from Southern Vietnam: Indication of Environmental Change during Crystallization
}

\author{
Doan Thi Anh Vu ${ }^{1,2}$, Alongkot Fanka ${ }^{1,3}$ (D) Abhisit Salam ${ }^{1}$ and Chakkaphan Sutthirat ${ }^{1, *}$ \\ 1 Department of Geology, Faculty of Science, Chulalongkorn University, Bangkok 10330, Thailand; \\ dtavu@hcmus.edu.vn (D.T.A.V.); alongkot.@@gmail.com (A.F.); masoesalam@gmail.com (A.S.) \\ 2 Faculty of Geology, University of Science, VNU-HCM, Ho Chi Minh City 008428, Vietnam \\ 3 Application Mineral and Petrology Special Task Force for Activating Research (AMP STAR), \\ Department of Geology, Faculty of Science, Chulalongkorn University, Bangkok 10330, Thailand \\ * Correspondence: chakkaphan.s@chula.ac.th
}

check for updates

Citation: Vu, D.T.A.; Fanka, A.; Salam, A.; Sutthirat, C. Variety of Iron Oxide Inclusions in Sapphire from Southern Vietnam: Indication of Environmental Change during Crystallization. Minerals 2021, 11, 241. https://doi.org/10.3390/ $\min 11030241$

Academic Editor:

Magdalena Dumańska-Słowik

Received: 5 December 2020

Accepted: 17 February 2021

Published: 26 February 2021

Publisher's Note: MDPI stays neutral with regard to jurisdictional claims in published maps and institutional affiliations.

Copyright: (c) 2021 by the authors. Licensee MDPI, Basel, Switzerland. This article is an open access article distributed under the terms and conditions of the Creative Commons Attribution (CC BY) license (https:/ / creativecommons.org/licenses/by/ $4.0 /)$.

\begin{abstract}
Sapphires from alluvial deposits associated with Cenozoic basalts in Southern Vietnam were collected for investigation of mineral inclusions. In this report, primary iron oxide inclusions were focused on, with detailed mineral chemistry using a Raman spectroscope and electron probe micro-analyzer. Consequently, a variety of iron oxide inclusions were recognized as wüstite, hercynite, and ilmenite. Ilmenite falling within an ilmenite-hematite series ranged in composition between $\mathrm{Il}_{24-30} \mathrm{He}_{36-38} \mathrm{Mt}_{35-40}$ and $\mathrm{Il}_{49-54} \mathrm{He}_{34-40} \mathrm{Mt}_{7-10}$, classified as titanomagnetite and titanohematite, respectively. Wüstite with nonstoichiometry, $\left(\mathrm{Fe}^{2+}{ }_{0.3-0.9}\right)\left(\mathrm{Ti}^{3+}<0.179 \mathrm{Al}^{3+} \leq 0.6 \mathrm{Cr}^{3+}<0.1 \mathrm{Fe}^{3+} \leq 0.46\right) \square_{\leq 0.23} \mathrm{O}$, was associated with hercynite inclusions, clearly indicating cogenetic sapphire formation. Wüstite and sapphire appear to have been formed from the breakdown reaction of hercynite (hercynite = sapphire+wüstite) within a reduction magma chamber. Titanohematite and titanomagnetite series might have crystallized during iron-titanium reequilibration via subsolidus exsolution under a slightly oxidized cooling process.
\end{abstract}

Keywords: sapphire; wüstite; hercynite; Cenozoic basalt

\section{Introduction}

Iron oxide minerals have been considered as a significant geothermometer for their host rocks [1-6]. Previous investigations of gem sapphire from Southern Vietnam, such as Dak Nong, Di Linh, and Binh Thuan deposits, have reported several iron oxide inclusions [7-9]. Most of these iron oxides were identified as ilmenite, magnetite-hercynite, and chromite-hercynite, using a scanning electron microscope-energy dispersive spectrometer (SEM-EDS) and an X-ray diffractometer (XRD) [8]. Moreover, Izokh et al. [7] reported an iron oxide inclusion, namely, Al-Ti-hematite, that was chemically analyzed by an electron probe micro-analyzer (EPMA); subsequently, they proposed that crystallization of host sapphire should relate to iron-rich syenitic melt and metasomatism between crustal rocks and contaminated basaltic melt in the Dak Nong deposit. Recently, Vu et al. [9] reported spinel and other unidentified oxide inclusions in sapphires from many deposits in Southern Vietnam which more details and further investigation are reported herein this manuscript. Besides, the crystallization of sapphire and related zircon from Southern Vietnam may occur in the lithospheric mantle which is related to carbonatite-dominant melts as a result of partial melting of a metasomatized lithospheric mantle source, at over $900{ }^{\circ} \mathrm{C}$ [10].

Although iron oxide inclusions were previously reported, their mineral chemistry has never been fully analyzed. This study was therefore designed to analyze most types of iron oxide inclusion in sapphire from Dak Nong, Di Linh, Binh Thuan, Krong Nang, and Pleiku. 


\section{Geological Setting}

Southern Vietnam geologically consists of a large-scale structure of the Da Lat active continental margin (Da Lat zone), Indosinian polyepisodic orogenic belt (Srepok orogenic belt), and Kontum massif [11], which is part of the Indosinian craton [12] (Figure 1). This region is composed of Archean-Proterozoic basement rocks, Early to Middle Paleozoic cover rocks, Jurassic sediments, late Mesozoic rocks, and Cenozoic basaltic rocks [12] (Figure 2). The basement rocks in this area are characterized by metamorphic complexes of granulites which are usually covered by volcanogenic sedimentary rocks, metamorphosed sedimentary rocks within greenschist facies of the Early to Middle Paleozoic, as well as sandstone, siltstone, and shale of Jurassic sedimentary formation. These basements and upper rocks are intruded by a number of late Mesozoic igneous rocks, including Triassic granite, granodiorite, and granosyenite (results of the Indosinian-Yangtze collision during the Permo-Triassic about 245-240 Ma) and Cretaceous diorite and granodiorite (result of the Paleo-Pacific plate subduction) [13-15]. Late Cenozoic basalts associated with sapphire deposits in Southern Vietnam have been mapped, overlining the older rock formations reported above.

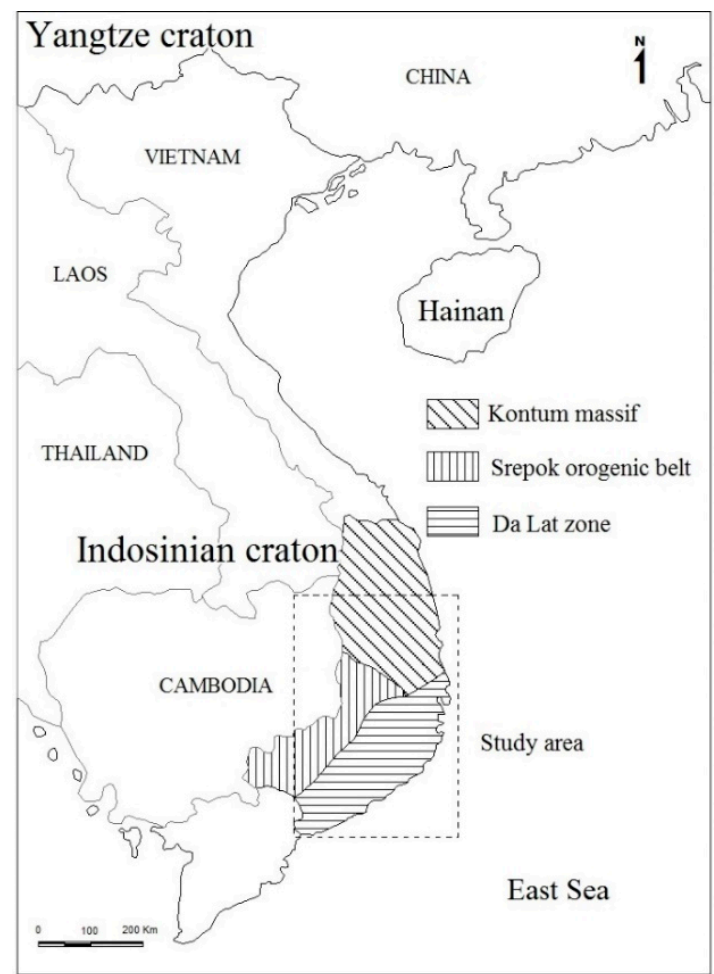

Figure 1. Geological map showing the study area and main tectonic terranes in Southern Vietnam, modified after Tri and Khuc [11] and Hoa et al. [12].

These Late Cenozoic basalts (Figure 2) are related to regional tectonic terranes, particularly after the end of East Sea opening in the Middle Miocene [16-18]. Paleo-Pacific oceanic crustal material, previously subducted into the lower mantle, was subsequently entrained into the Hainan plume which was the main cause of basaltic magmatism in Southern Vietnam [19]. According to Hoang and Flower [20], these volcanic activities were extended more than $100 \mathrm{~km}$ in diameter with a thickness up to several hundred meters and covered a total area of approximately $23,000 \mathrm{~km}^{2}$. The centers of volcanism appear to have developed during two main eruptive episodes. The early phases generated mainly quartz and olivine tholeiites with rare alkali basalt whereas the later phases produced olivine tholeiite, alkali basalt, basanite, and rare nephelinite. Tholeiite eruptions occurred significantly in the centers associated with extensional rifting. On the other hand, alkali basalt, olivine tholeiite, and basanite appear to have erupted along the conjugate strike-slip faults [18]. It 
should be noted that sapphire and zircon occurrences mainly discovered in Quaternary and Upper-Pleistocene alluvial deposits derived from the alkali basalts [8-10,21]. They would originate in the deep-seated formations before being transported as megacryst onto the Earth's surface by alkali basaltic magmas.

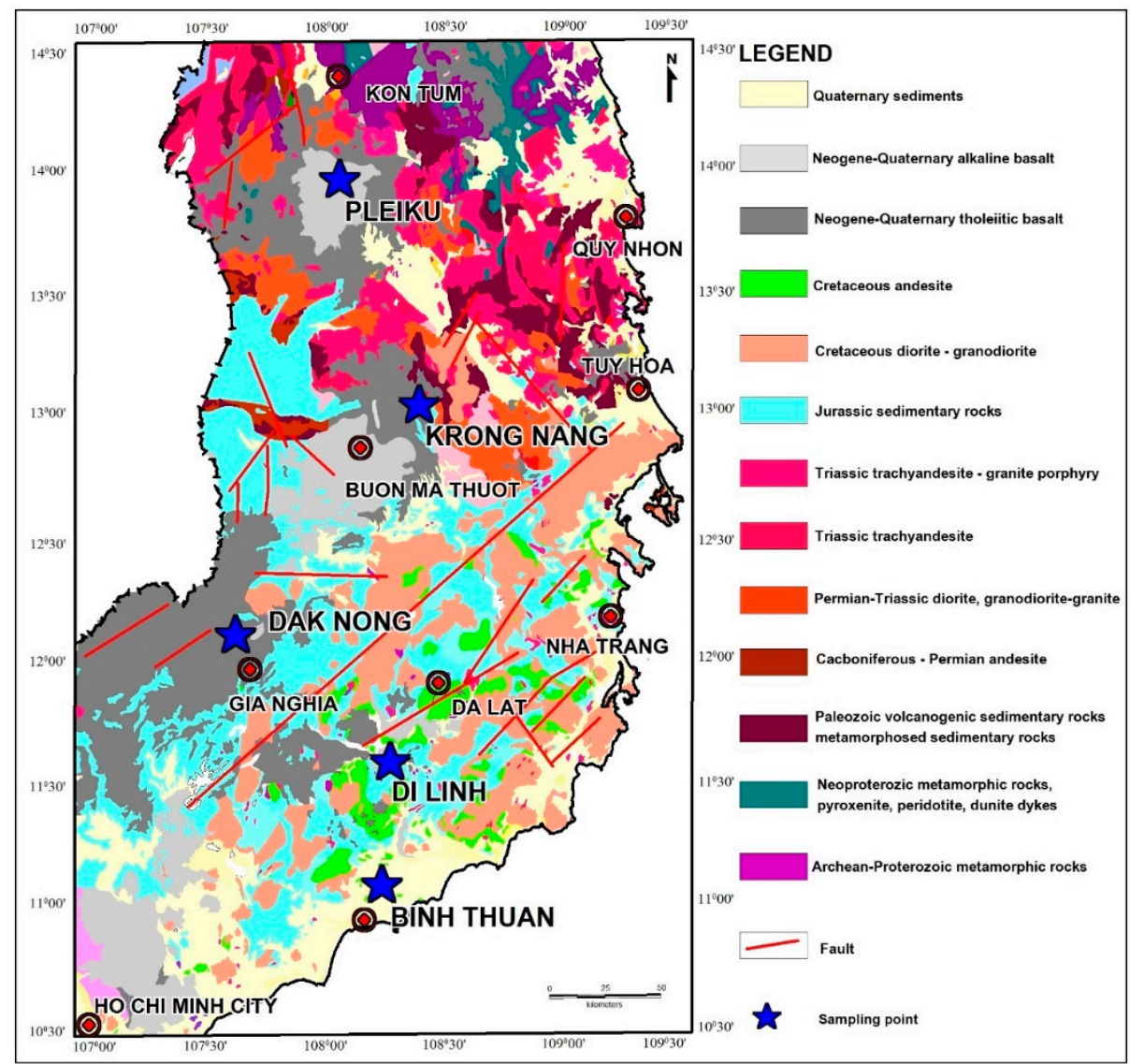

Figure 2. Geological map showing alluvial sapphire deposits and associated Cenozoic basalts underlain by the older rock formations in Southern Vietnam, modified after Hoa et al. [12].

\section{Materials and Methods}

Sapphire collections of about $5 \mathrm{~mm}$ crystals were sampled from Dak Nong, Di Linh, Binh Thuan, Krong Nang, and Pleiku in Southern Vietnam (see Figure 2 and Table 1 and Table S1). The sapphire crystals were detected for metallic opaque inclusions for further study. After being mounted in epoxy, they were ground by a diamond wheel until inclusions were exposed; subsequently, they were polished using $6 \mu \mathrm{m}, 3 \mu \mathrm{m}$, and $1 \mu \mathrm{m}$ diamond pastes. These inclusions were initially characterized using a laser Raman spectroscope, inVia model, Renishaw equipped with a Leica optical microscope at the Gem and Jewelry Institute of Thailand (Public Organization) (GIT). The laser beam was generally set at about $5 \mu \mathrm{m}$ with standard conditions including $532 \mathrm{~nm}$ radiation of the NIR diode laser emitting at $785 \mathrm{~nm}$ and power of $15.7 \mathrm{~mW}$ (about $5 \mathrm{~mW}$ on the sample), at a resolution of approximately $0.5 \mathrm{~cm}^{-1}$. It should be noted that Raman patterns of iron minerals may be transformed rapidly by laser induction, leading to the modification of Raman shift, and the same effect can also be induced by the natural processes such as oxidation, recrystallization, order-disorder transitions (cation redistribution), phase transition, or decomposition [22-24]. Therefore, low laser power $(0.5 \mathrm{~mW})$ and normal operation power $(5 \mathrm{~mW})$ on the sample were applied in this study to observe the Raman pattern and its alteration in wüstite inclusions because its structure is sensitive to laser power. However, ilmenite and hercynite spinel were analyzed using normal operation laser power of $5 \mathrm{~mW}$, as suggested by Wang et al. [25]. Each spectrum was recorded within 
the spectral range of $200 \mathrm{~cm}^{-1}$ to $1500 \mathrm{~cm}^{-1}$ for $20 \mathrm{~s}$ of exposure time, 6 accumulation, and $50 \times$ magnification, at a laboratory temperature of about $22^{\circ} \mathrm{C}$.

After identification using the Raman technique, these inclusion samples were carbon coated prior to major and minor analyses using an electron probe microanalyzer (EPMA, JEOL model JXA-8100) at the Department of Geology, Faculty of Science, Chulalongkorn University. The analytical condition was set at $15 \mathrm{kV}$ acceleration voltages with about $24 \mathrm{nA}$ probe current. Appropriate standards and analytical crystals were selected for analyses with $30 \mathrm{~s}$ for peak counts and background counts of each element before automatic ZAF correction, requested for three main effects (i.e., atomic number, absorption, and fluorescence excitation) influencing spectroscopic analysis of characteristic X-rays, was applied to report in oxide contents. Finally, atomic proportions of these analyses were recalculated on the basis of proper oxygen in their formular before $\mathrm{Fe}^{2+}$ and $\mathrm{Fe}^{3+}$ ratios were estimated accordingly using the equation of Droop [26].

\section{Results}

Although all of the studied iron oxide minerals looked similarly black opaque crystals, their morphological affinities were classified into two groups, octahedral and rhombohedral shapes, indicating significantly primary inclusions. Octahedral iron oxides formed as single and aggregate crystals ranging in size from 20-500 $\mu \mathrm{m}$ (Figure 3). In contrast, rhombohedral iron oxides occurred typically as single crystals, about $80-600 \mu \mathrm{m}$ long, and 40-300 $\mu \mathrm{m}$ wide (Figure 4). Raman spectroscopic identification of these iron oxide inclusions is reported below.

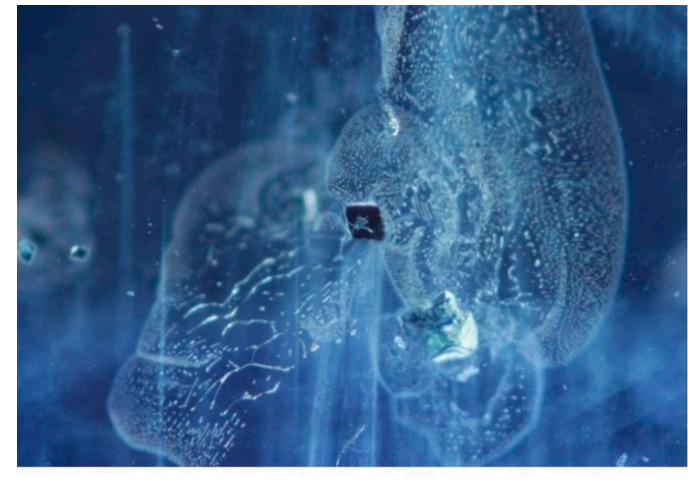

(a)

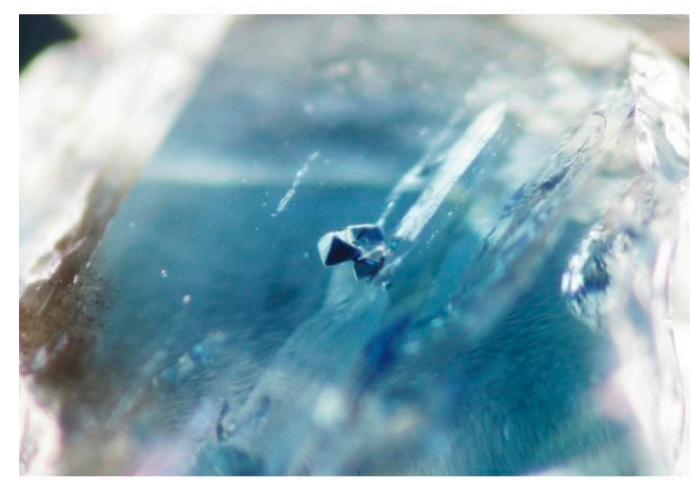

(c)

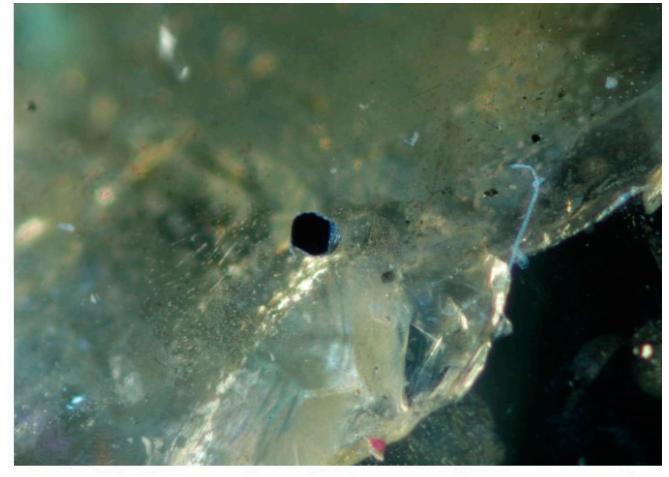

(b)

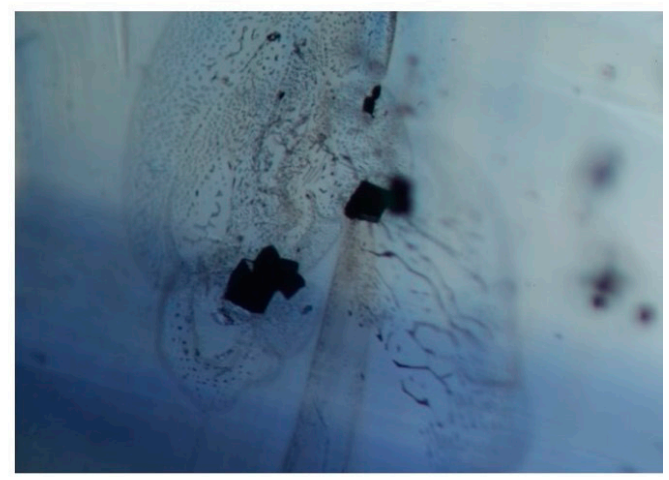

(d)

Figure 3. Photomicrographs taken with dark field illumination, magnified $50 \times$, showing: (a) a small octahedral wüstite inclusion in sapphire (sample DL50) from Di Linh; (b) a small octahedral hercynite inclusion in sapphire (sample KN43) from Krong Nang; (c) aggregate crystals of octahedral wüstite inclusions in sapphire (sample GL27) from Pleiku, and (d) aggregate crystals of octahedral hercynite inclusions in sapphire (sample DN16) from Dak Nong. 


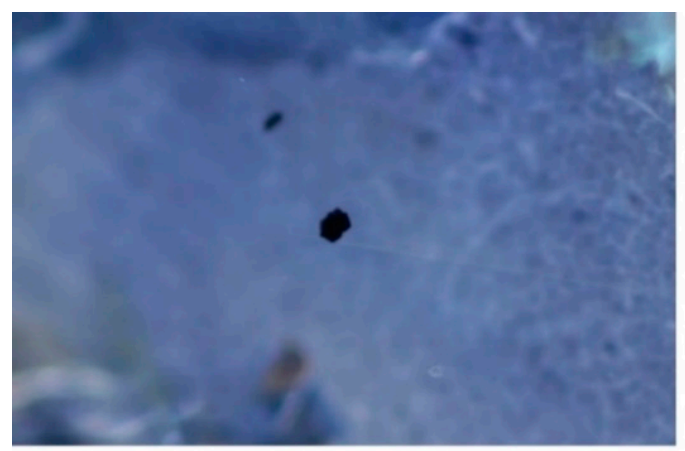

(a)

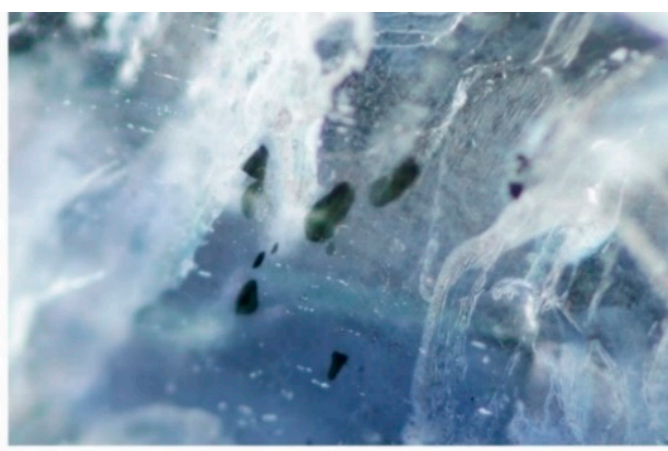

(b)

Figure 4. Photomicrographs taken with dark field illumination, magnified 50×, showing rhombohedral ilmenite inclusions: (a) a single crystal (sample DL85 from Di Linh) as reported by Vu et al. [9] and (b) several single crystals (sample GL25 from Pleiku).

Three distinctive types were recognized by Raman spectra, including wüstite, hercynite, and ilmenite groups. In comparison with morphological features, wüstite and hercynite inclusions typically showed octahedral shape in forms of single and aggregate crystals. Moreover, wüstite-hercynite composite inclusions (Figure 5) could be found. On the other hand, ilmenite inclusions were mostly characterized by rhombohedral single crystals.

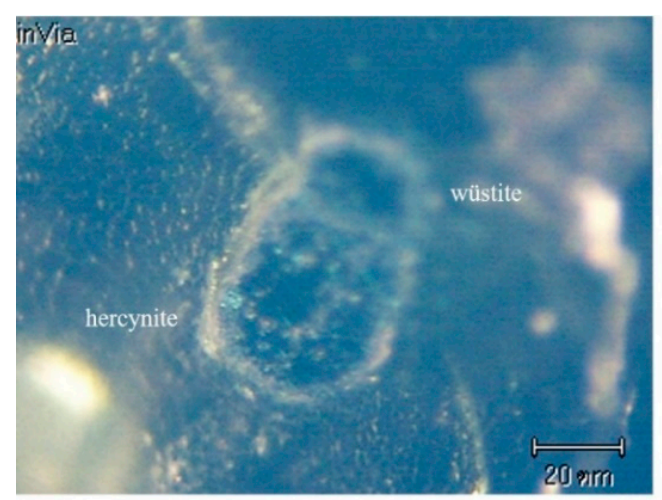

(a)

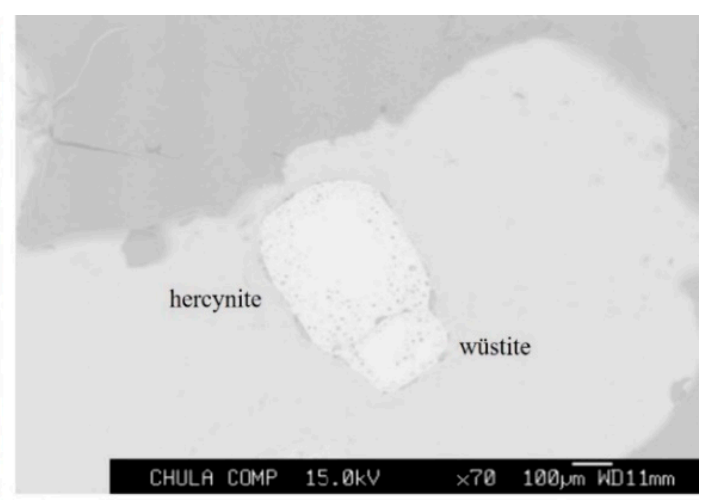

(b)

Figure 5. (a) Photomicrographs and (b) black-scattered electron images (BSIs) showing hercynite-wüstite compositee inclusion found in sapphire (sample PT55) from Binh Thuan.

At the low laser power $(0.5 \mathrm{~mW})$, all studied wüstite inclusions clearly showed only a sharp peak at $670 \mathrm{~cm}^{-1}$, which closely resembled magnetite spectra (Figure 6a). On the other hand, at high laser power $(5 \mathrm{~mW})$, some wüstite inclusions (e.g., DL50, DL56, PT17) showed characteristic patterns of magnetite (weaker peak in the range of $650-670 \mathrm{~cm}^{-1}$ ), hematite with higher intensity peaks at 218, 285, and 388, and wüstite assigned by the 595 $\mathrm{cm}^{-1}$ peak, as suggested by Hanesch [23] (Figure 6b). These spectra were matched well with wüstite spectra observed at low and high laser powers reported by Faria et al. [22]. In addition, some wüstite inclusions showed a broader band in the $650-670 \mathrm{~cm}^{-1}$ region (strongest peak at approximately $667 \mathrm{~cm}^{-1}$ ) of magnetite with higher intensity (Figure 6c). 


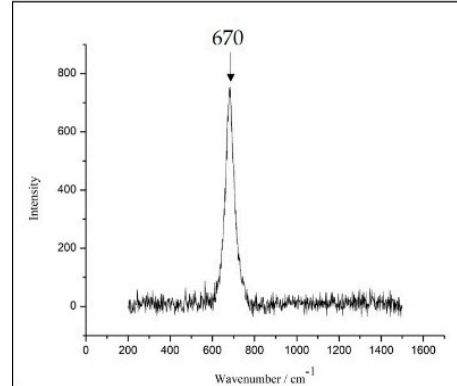

(a)

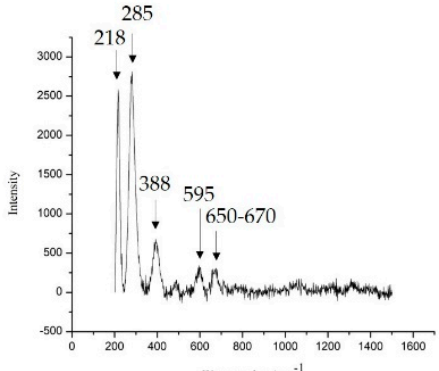

(b)

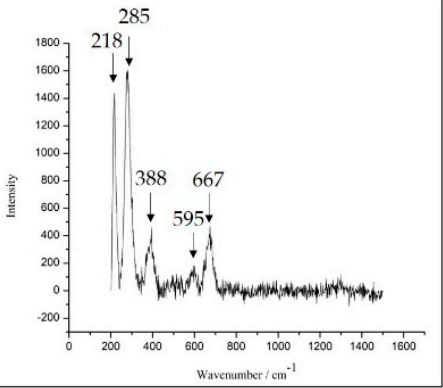

(c)

Figure 6. Raman spectra of wüstite inclusion observed at different laser powers, $0.5 \mathrm{~mW}$ (a) and $5 \mathrm{~mW}$ (b,c); sample DL50 from Di Linh (a,b) and sample PT49 from Binh Thuan (c).

The Raman spectrum of hercynite is representatively displayed in Figure 7. The spectrum yielded a strong band at $753 \mathrm{~cm}^{-1}$ and a weak peak at $701 \mathrm{~cm}^{-1}$ which indicate the vibration of $\mathrm{AlO}_{4}$ tetrahedra, characteristic of a spinel structure, as suggested by Cynn et al. [27]. This spectrum was similar to the hercynite spinel reported by Ospitali et al. [28].

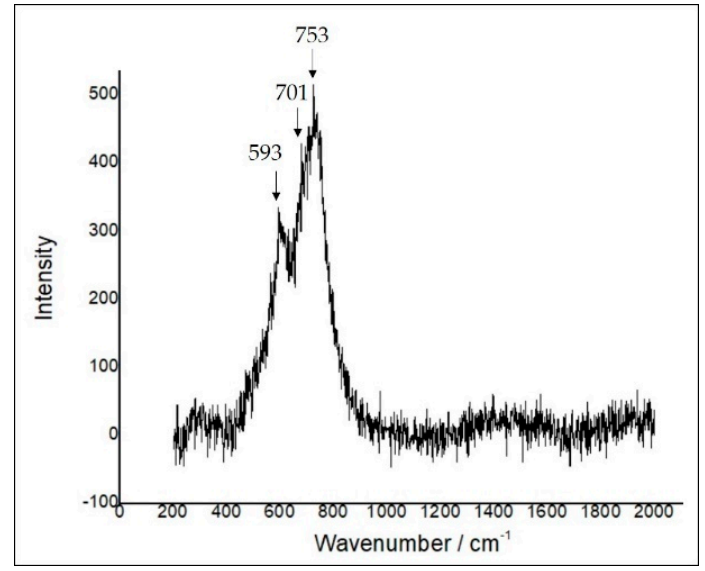

Figure 7. Raman spectrum, measured at $5 \mathrm{~mW}$ laser power, of hercynite spinel inclusion in sapphire (sample KN43) from Krong Nang.

An ilmenite inclusion showed Raman characteristics of mixture minerals of which Raman peaks were recognized at 299, 498, and $683 \mathrm{~cm}^{-1}$ (Figure 8a). The highest intensity peak, $683 \mathrm{~cm}^{-1}$, was due to ilmenite, as suggested by Wang et al. [25], whereas 299 and $498 \mathrm{~cm}^{-1}$ peaks were caused by hematite [22]. Additionally, some ilmenite inclusions revealed broadened peaks at 220,293 , and $613 \mathrm{~cm}^{-1}$ which matched well with the hematite's pattern; moreover, an additional characteristic peak of ilmenite was present around $683 \mathrm{~cm}^{-1}$ as well as weak peaks at 399,600, and $1298 \mathrm{~cm}^{-1}$, likely indicating oxidized titanomagnetite (Figure 8b) [24,25]. These different Raman patterns indicated that ilmenite inclusions in this study may have a variety of chemical compositions. 


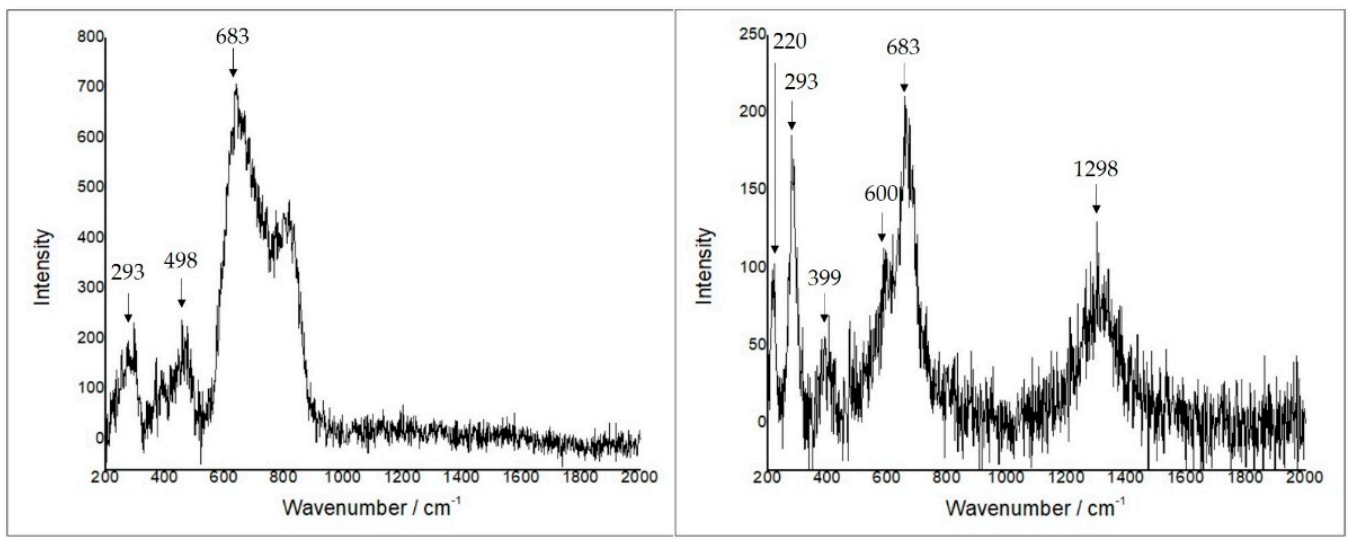

(a)

(b)

Figure 8. Raman spectra, measured at $5 \mathrm{~mW}$ laser power, of ilmenite inclusions showing different patterns as reported in the main text; (a) sample DL85 from Di Linh and (b) sample GL25 from Pleiku.

Mineral chemistry of these iron oxide inclusions, based on the EPMA, clearly supported Raman spectroscopic identification. Representative EPMA analyses of each iron oxide type are shown in Tables 1-3.

Table 1. Representative EPMA analyses of wüstite inclusions in sapphire from Southern Vietnam.

\begin{tabular}{|c|c|c|c|c|c|c|c|c|c|c|}
\hline \multirow{4}{*}{$\begin{array}{c}\text { Mineral } \\
\text { Phase } \\
\text { Analysis } \\
(w t \%)\end{array}$} & \multicolumn{6}{|c|}{ Wüstite (FeO)-Hercynite } & \multicolumn{4}{|c|}{ Wüstite (FeO) } \\
\hline & \multirow{3}{*}{$\begin{array}{c}\text { Dak Nong } \\
\text { DN74 }\end{array}$} & \multicolumn{4}{|c|}{ Southern Vietnam } & \multirow{3}{*}{$\begin{array}{l}\text { Pleiku } \\
\text { GL27 } \\
\end{array}$} & \multicolumn{3}{|c|}{ Southern Vietnam } & \multirow{3}{*}{ Germany } \\
\hline & & Di Linh & \multirow{2}{*}{$\begin{array}{c}\text { Krong Nang } \\
\text { KN19* }\end{array}$} & \multicolumn{2}{|c|}{ Binh Thuan } & & \multicolumn{2}{|c|}{ Di Linh } & \multirow{2}{*}{$\begin{array}{c}\text { Binh Thuan } \\
\text { PT17a }\end{array}$} & \\
\hline & & DL14 * & & PT49 & PT55a & & DL50 * & DL56 & & \\
\hline $\mathrm{SiO}_{2}$ & 0.03 & 0.00 & 0.06 & 0.44 & 0.44 & 0.34 & 0.02 & 0.15 & 0.27 & 0.85 \\
\hline $\mathrm{TiO}_{2}$ & 0.84 & 1.53 & 1.24 & 5.89 & 0.38 & 0.00 & 2.33 & 3.25 & 0.22 & 0.04 \\
\hline $\mathrm{Al}_{2} \mathrm{O}_{3}$ & 9.85 & 6.66 & 11.84 & 12.88 & 19.28 & 11.39 & 0.21 & 0.27 & 0.12 & 0.29 \\
\hline $\mathrm{Cr}_{2} \mathrm{O}_{3}$ & 0.01 & 0.04 & 0.03 & 0.03 & 0.00 & 0.03 & 0.02 & 0.04 & 0.03 & na \\
\hline $\mathrm{FeO}$ & 88.13 & 89.29 & 86.30 & 77.44 & 77.44 & 86.25 & 96.45 & 95.31 & 95.46 & 91.94 \\
\hline $\mathrm{MnO}$ & 0.20 & 1.49 & 0.18 & 2.25 & 2.25 & 0.00 & 0.41 & 0.35 & 1.81 & 0.14 \\
\hline $\mathrm{MgO}$ & 0.35 & 0.29 & 0.37 & 0.20 & 0.23 & 0.00 & 1.36 & 0.40 & 0.88 & 0.12 \\
\hline $\mathrm{ZnO}$ & 0.67 & 0.36 & 0.51 & 0.30 & 0.25 & 0.07 & 0.00 & 0.00 & 0.00 & na \\
\hline $\mathrm{CaO}$ & 0.00 & 0.00 & 0.00 & 0.00 & 0.00 & 0.00 & 0.00 & 0.29 & 0.00 & 0.14 \\
\hline $\mathrm{NiO}$ & 0.02 & 0.00 & 0.10 & 0.01 & 0.02 & 0.05 & 0.03 & 0.00 & 0.00 & na \\
\hline Total & 100.1 & 99.66 & 100.52 & 99.47 & 99.90 & 98.12 & 100.8 & 100.1 & 98.78 & 93.52 \\
\hline \multicolumn{11}{|c|}{ Formula $4(\mathrm{O})$} \\
\hline $\mathrm{Si}$ & 0.001 & 0.000 & 0.002 & 0.002 & 0.002 & 0.015 & 0.001 & 0.007 & 0.013 & 0.043 \\
\hline $\mathrm{Ti}$ & 0.027 & $\begin{array}{l}0.051 \\
0.000\end{array}$ & 0.039 & 0.178 & 0.011 & 0.000 & 0.081 & 0.113 & 0.008 & 0.002 \\
\hline $\mathrm{Al}$ & 0.496 & 0.346 & 0.580 & 0.612 & 0.892 & 0.577 & 0.011 & 0.015 & 0.007 & 0.017 \\
\hline $\mathrm{Cr}$ & 0.000 & 0.001 & 0.001 & 0.001 & 0.000 & 0.001 & 0.001 & 0.001 & 0.001 & \\
\hline $\mathrm{Fe}^{2+}$ & 3.147 & 3.291 & 3.001 & 2.604 & 2.541 & 1.507 & 3.708 & 3.681 & 1.962 & 3.863 \\
\hline $\mathrm{Mn}$ & 0.007 & 0.056 & 0.006 & 0.081 & 0.075 & 0.000 & 0.016 & 0.014 & 0.073 & 0.006 \\
\hline $\mathrm{Mg}$ & 0.022 & 0.019 & 0.023 & 0.009 & 0.011 & 0.000 & 0.093 & 0.027 & 0.062 & 0.009 \\
\hline $\mathrm{Zn}$ & 0.021 & 0.012 & 0.016 & 0.000 & 0.009 & 0.002 & 0.000 & 0.000 & 0.000 & \\
\hline $\mathrm{Ca}$ & 0.000 & 0.000 & 0.000 & 0.024 & 0.000 & 0.000 & 0.000 & 0.014 & 0.000 & 0.008 \\
\hline $\mathrm{Ni}$ & 0.001 & 0.000 & 0.000 & 0.003 & 0.000 & 0.002 & 0.001 & 0.000 & 0.000 & \\
\hline Total * & 3.723 & 3.776 & 3.660 & 3.514 & 3.541 & 3.697 & 3.912 & 3.872 & 3.975 & 3.947 \\
\hline$\Sigma \mathrm{R}^{2+}$ & 3.198 & 3.378 & 3.046 & 2.721 & 2.636 & 1.511 & 3.818 & 3.736 & 2.097 & 3.886 \\
\hline$\Sigma \mathrm{R}^{3+}$ & 0.524 & 0.397 & 0.622 & 0.793 & 0.545 & 0.9 .5 & 0.093 & 0.135 & 0.028 & 0.062 \\
\hline \% wüstite & 86 & 89 & 83 & 77 & 85 & 84 & 98 & 96 & 99 & 98 \\
\hline $\begin{array}{c}\% \\
\text { hercynite }\end{array}$ & 14 & 11 & 17 & 23 & 15 & 16 & 2 & 4 & 1 & 2 \\
\hline
\end{tabular}

Note: * previously reported by $\mathrm{Vu}$ et al. [9]; ${ }^{1}$ Seifert et al. [29]; $\mathrm{na}=$ not analyzed; $\sum \mathrm{R}^{2+}=\mathrm{Fe}^{2+}+\mathrm{Mn}+\mathrm{Mg}+\mathrm{Zn}+\mathrm{Ca}+\mathrm{Ni} ; \sum \mathrm{R}^{3+}=\mathrm{Si}+\mathrm{Ti}+$ $\mathrm{Al}+\mathrm{Cr}+\mathrm{Fe}^{3+}$. 
Table 2. Representative EPMA analyses of hercynite spinel inclusions in sapphire from Southern Vietnam.

\begin{tabular}{|c|c|c|c|c|c|c|c|c|c|}
\hline \multirow{2}{*}{$\begin{array}{l}\text { Mineral Phase } \\
\text { Analysis (wt\%) }\end{array}$} & \multicolumn{2}{|c|}{ Dak Nong } & \multirow{2}{*}{$\frac{\text { Di Linh }}{\text { DL62 * }}$} & \multicolumn{2}{|c|}{ Krong Nang } & \multicolumn{3}{|c|}{ Binh Thuan } & \multirow{2}{*}{$\begin{array}{r}\text { Pleiku } \\
\text { GL07 }\end{array}$} \\
\hline & DN12 * & DN16 * & & KN20b & KN43 & PT07 & PT17b & PT55b & \\
\hline $\mathrm{SiO}_{2}$ & 0.00 & 0.00 & 0.01 & 0.00 & 0.00 & 0.02 & 0.00 & 0.00 & 0.02 \\
\hline $\mathrm{TiO}_{2}$ & 0.12 & 0.08 & 0.70 & 0.00 & 0.00 & 0.00 & 0.27 & 0.00 & 0.32 \\
\hline $\mathrm{Al}_{2} \mathrm{O}_{3}$ & 60.99 & 60.93 & 60.76 & 61.07 & 60.68 & 61.12 & 61.02 & 60.94 & 60.02 \\
\hline $\mathrm{Cr}_{2} \mathrm{O}_{3}$ & 0.00 & 0.00 & 0.09 & 0.07 & 0.02 & 0.01 & 0.00 & 0.00 & 0.00 \\
\hline $\mathrm{FeO}$ & 33.99 & 34.18 & 33.87 & 33.84 & 32.24 & 33.40 & 35.38 & 34.62 & 33.87 \\
\hline $\mathrm{MnO}$ & 0.00 & 0.89 & 0.34 & 0.81 & 0.28 & 0.06 & 1.47 & 0.46 & 0.98 \\
\hline $\mathrm{MgO}$ & 4.02 & 4.29 & 4.62 & 4.61 & 5.26 & 4.55 & 0.56 & 4.07 & 5.23 \\
\hline $\mathrm{ZnO}$ & 0.12 & 0.38 & 0.18 & 0.00 & 0.63 & 0.67 & 0.01 & 0.18 & 0.00 \\
\hline $\mathrm{CaO}$ & 0.06 & 0.00 & 0.00 & 0.00 & 0.00 & 0.00 & 0.00 & 0.01 & 0.00 \\
\hline $\mathrm{NiO}$ & 0.00 & 0.00 & 0.00 & 0.03 & 0.00 & 0.09 & 0.10 & 0.00 & 0.00 \\
\hline Total & 99.29 & 100.74 & 100.58 & 100.43 & 98.81 & 99.91 & 99.81 & 100.27 & 100.45 \\
\hline \multicolumn{10}{|l|}{ Formula 32(O) } \\
\hline $\mathrm{Si}$ & 0.000 & 0.000 & 0.002 & 0.000 & 0.000 & 0.003 & 0.003 & 0.000 & 0.005 \\
\hline $\mathrm{Ti}$ & 0.020 & 0.014 & 0.117 & 0.000 & 0.046 & 0.000 & 0.046 & 0.000 & 0.054 \\
\hline $\mathrm{Al}$ & 16.134 & 15.975 & 15.886 & 16.003 & 16.433 & 16.071 & 16.433 & 16.040 & 15.763 \\
\hline $\mathrm{Cr}$ & 0.000 & 0.000 & 0.016 & 0.012 & 0.000 & 0.002 & 0.000 & 0.000 & 0.000 \\
\hline $\mathrm{Fe}^{3+}$ & 0.000 & 0.000 & 0.000 & 0.000 & 0.000 & 0.000 & 0.000 & 0.000 & 0.157 \\
\hline $\mathrm{Fe}^{2+}$ & 6.380 & 6.359 & 6.285 & 6.292 & 6.762 & 6.232 & 6.762 & 6.466 & 6.155 \\
\hline $\mathrm{Mn}$ & 0.000 & 0.168 & 0.064 & 0.152 & 0.285 & 0.012 & 0.285 & 0.087 & 0.186 \\
\hline $\mathrm{Mg}$ & 1.344 & 1.421 & 1.529 & 1.529 & 0.191 & 1.514 & 0.191 & 1.355 & 1.739 \\
\hline $\mathrm{Zn}$ & 0.020 & 0.062 & 0.030 & 0.000 & 0.002 & 0.111 & 0.002 & 0.030 & 0.000 \\
\hline $\mathrm{Ca}$ & 0.015 & 0.000 & 0.000 & 0.000 & 0.000 & 0.000 & 0.000 & 0.002 & 0.000 \\
\hline $\mathrm{Ni}$ & 0.000 & 0.000 & 0.000 & 0.006 & 0.018 & 0.017 & 0.018 & 0.000 & 0.000 \\
\hline Total * & 23.913 & 23.999 & 23.929 & 23.993 & 23.737 & 23.960 & 23.737 & 23.980 & 24.059 \\
\hline$\Sigma \mathrm{R}^{2+}$ & 7.759 & 8.010 & 7.907 & 7.979 & 7.259 & 7.885 & 7.259 & 7.940 & 8.080 \\
\hline$\Sigma \mathrm{R}^{3+}$ & 16.154 & 15.989 & 16.022 & 16.014 & 16.479 & 16.076 & 16.479 & 16.040 & 15.979 \\
\hline
\end{tabular}

Note: ${ }^{*}$ previously reported by Vu et al. [9]. $\mathrm{Fe}^{2+}$ and $\mathrm{Fe}^{3+}$ were recalculated from total $\mathrm{FeO}$ after the method of Droop [26]. $\Sigma \mathrm{R}^{2+}=\mathrm{Fe}^{2+}+$ $\mathrm{Mn}+\mathrm{Mg}+\mathrm{Zn}+\mathrm{Ca}+\mathrm{Ni} . \Sigma \mathrm{R}^{3+}=\mathrm{Si}+\mathrm{Ti}+\mathrm{Al}+\mathrm{Cr}+\mathrm{Fe}^{3+}$.

Wüstite varied in composition from about $77-97 \% \mathrm{FeO},<20 \% \mathrm{Al}_{2} \mathrm{O}_{3}$, and $<7 \% \mathrm{TiO}_{2}$ with traces of $\mathrm{MnO}$ (Table 1). Based on these compositions, it was mostly characterized by a non-stoichiometric formula. The compositions are similar to wüstite that formed under strongly reducing conditions in Rumburk granite in Germany (Table 1). Recalculated atomic proportions show that the hercynite component (ranging from 1 to $23 \%$ ) may play an important role in the wüstite composition (Seifert et al. [29], Table 1). Based on the procedure of Smyth et al. [30], the average chemical formula can be expressed for nonstoichiometric wüstite as $\left(\mathrm{Fe}^{2+}{ }_{0.3-0.9}\right)\left(\mathrm{Ti}^{3+}<0.179 \mathrm{Al}^{3+} \leq 0.6 \mathrm{Cr}^{3+}<0.1 \mathrm{Fe}^{3+} \leq 0.46\right) \square \leq 0.23 \mathrm{O}$, where $\square$ is deficiency of Fe.

Hercynite spinel inclusions presented narrow compositional ranges of $60-62 \% \mathrm{Al}_{2} \mathrm{O}_{3}$, $32-36 \% \mathrm{FeO}$, and $\leq 5.3 \% \mathrm{MgO}$ (Table 2). Smith et al. [8] previously reported the composition of hercynite inclusions in Di Linh and Binh Thuan sapphires, based on SEM-EDS analyses, varying between chromite-hercynite and magnetite-hercynite series. EPMA analyses of hercynite inclusions in this study showed an almost pure hercynite composition $\left(\mathrm{Fe}^{2+}{ }_{6.0-6.8} \mathrm{Mn}^{2+}<0.3 \mathrm{Mg}^{2+}<1.8 \mathrm{Zn}^{2+}<0.2 \mathrm{Ca}_{<0.1}\right)\left(\mathrm{Ti}^{3+}{ }_{<0.3} \mathrm{Al}^{3+}{ }_{15.8-16.4} \mathrm{Fe}^{3+}<0.2\right) \mathrm{O}_{32}$ (Figure 9). It should be noted again that some hercynite was found in close association with wüstites in sapphire from Binh Thuan (sample PT55, as shown in Figure 5, with EPMA analyses presented in Tables 1 and 2). 
Table 3. Representative EPMA analyses of ilmenite inclusions in sapphire from Southern Vietnam.

\begin{tabular}{|c|c|c|c|c|c|c|}
\hline \multirow{3}{*}{$\begin{array}{l}\text { Mineral Phase } \\
\text { Analysis (wt } \%)\end{array}$} & \multicolumn{4}{|c|}{ Titanohematite } & \multirow{2}{*}{\multicolumn{2}{|c|}{$\begin{array}{c}\text { Titanomagnetite } \\
\text { Pleiku }\end{array}$}} \\
\hline & \multirow{2}{*}{$\frac{\text { Dak Nong }}{\text { DN39* }}$} & \multicolumn{2}{|c|}{ Di Linh } & \multirow{2}{*}{$\begin{array}{c}\text { Pleiku } \\
\text { GL28 }\end{array}$} & & \\
\hline & & DL23 * & DL85 * & & GL25 & GL86 \\
\hline $\mathrm{SiO}_{2}$ & 0.11 & 0.10 & 0.00 & 0.08 & 0.17 & 0.09 \\
\hline $\mathrm{TiO}_{2}$ & 34.94 & 37.08 & 33.77 & 34.89 & 18.48 & 20.27 \\
\hline $\mathrm{Al}_{2} \mathrm{O}_{3}$ & 0.02 & 1.50 & 0.58 & 1.14 & 3.25 & 2.37 \\
\hline $\mathrm{Cr}_{2} \mathrm{O}_{3}$ & 0.00 & 0.04 & 0.12 & 0.05 & 0.00 & 0.04 \\
\hline $\mathrm{FeO}$ & 61.43 & 61.58 & 63.48 & 63.00 & 76.12 & 74.54 \\
\hline $\mathrm{MnO}$ & 0.14 & 0.06 & 0.05 & 0.09 & 0.64 & 0.28 \\
\hline $\mathrm{MgO}$ & 0.00 & 0.18 & 0.64 & 0.07 & 0.06 & 0.46 \\
\hline $\mathrm{ZnO}$ & 0.80 & 0.00 & 0.00 & 0.00 & 0.00 & 0.00 \\
\hline $\mathrm{CaO}$ & 0.00 & 0.01 & 0.00 & 0.00 & 0.04 & 0.00 \\
\hline $\mathrm{NiO}$ & 0.02 & 0.00 & 0.03 & 0.06 & 0.00 & 0.05 \\
\hline Total & 97.46 & 100.54 & 98.67 & 99.38 & 99.11 & 98.10 \\
\hline \multicolumn{7}{|l|}{ Formula $6(\mathrm{O})$} \\
\hline $\mathrm{Si}$ & 0.006 & 0.006 & 0.000 & 0.005 & 0.000 & 0.005 \\
\hline $\mathrm{Ti}$ & 1.499 & 1.514 & 1.437 & 1.463 & 1.310 & 0.931 \\
\hline $\mathrm{Al}$ & 0.001 & 0.096 & 0.039 & 0.075 & 0.435 & 0.171 \\
\hline $\mathrm{Cr}$ & 0.000 & 0.002 & 0.005 & 0.002 & 0.001 & 0.002 \\
\hline $\mathrm{Fe}^{3+}$ & 1.318 & 1.168 & 1.430 & 1.319 & 1.265 & 3.808 \\
\hline $\mathrm{Fe}^{2+}$ & 1.612 & 1.629 & 1.573 & 1.618 & 1.411 & 0.014 \\
\hline Mn & 0.007 & 0.003 & 0.002 & 0.004 & 0.042 & 0.042 \\
\hline $\mathrm{Mg}$ & 0.000 & 0.014 & 0.054 & 0.006 & 0.006 & 0.000 \\
\hline $\mathrm{Zn}$ & 0.049 & 0.000 & 0.000 & 0.000 & 0.000 & 0.000 \\
\hline $\mathrm{Ca}$ & 0.000 & 0.000 & 0.000 & 0.000 & 0.000 & 0.002 \\
\hline $\mathrm{Ni}$ & 0.001 & 0.000 & 0.001 & 0.003 & 0.000 & 0.005 \\
\hline Total * & 4.494 & 4.431 & 4.541 & 4.494 & 4.471 & 4.977 \\
\hline$\Sigma \mathrm{R}^{2+}$ & 1.668 & 1.646 & 1.630 & 1.630 & 1.459 & 1.512 \\
\hline$\Sigma \mathrm{R}^{3+}$ & 2.825 & 2.785 & 2.911 & 2.864 & 3.012 & 3.465 \\
\hline \%llmenite & 53.1 & 54.4 & 49.4 & 51.1 & 24.0 & 29.9 \\
\hline \%magnetite & 6.8 & 6.9 & 8.3 & 9.5 & 40.0 & 34.5 \\
\hline \%hematite & 38.7 & 33.7 & 39.8 & 35.5 & 35.5 & 37.9 \\
\hline
\end{tabular}

Note: * previously reported by Vu et al. [9]. $\mathrm{Fe}^{2+}$ and $\mathrm{Fe}^{3+}$ were recalculated from total $\mathrm{FeO}$ after the method of Droop [26]. $\Sigma \mathrm{R}^{2+}=\mathrm{Fe}^{2+}+$ $\mathrm{Mn}+\mathrm{Mg}+\mathrm{Zn}+\mathrm{Ca}+\mathrm{Ni} . \Sigma \mathrm{R}^{3+}=\mathrm{Si}+\mathrm{Ti}+\mathrm{Al}+\mathrm{Cr}+\mathrm{Fe}^{3+}$.

Ilmenite inclusions yielded EPMA analyses, as summarized in Table 3. Their compositions ranged from about $34-37 \% \mathrm{TiO}_{2}, 61-64 \% \mathrm{FeO}$, and $\leq 1.5 \% \mathrm{Al}_{2} \mathrm{O}_{3}$. However, two ilmenite inclusions (GL25 and GL86) in Pleiku sapphire show higher Fe contents (75-76\% $\mathrm{FeO})$ with lower Ti contents $\left(19-20 \% \mathrm{TiO}_{2}\right)$. Ternary plots of the $\mathrm{FeO}-\mathrm{Fe}_{2} \mathrm{O}_{3}-\mathrm{TiO}_{2}$ system (Figure 10) showed clearly that most ilmenite inclusions fall within ilmenite-hematite series in which GL25 and GL86 samples move towards the hematite component. Consequently, their recalculated atomic proportions yielded different end-member ratios of ilmenite-hematite-magnetite as $\mathrm{Il}_{24-30} \mathrm{He}_{36-38} \mathrm{Mt}_{35-40}$ and $\mathrm{Il}_{49-54} \mathrm{He}_{34-40} \mathrm{Mt}_{7-10}$, which can be grouped into titanomagnetite and titanohematite, respectively (Table 3 ).

\section{Discussion}

\subsection{Classification of Iron Oxide Inclusions}

Based on morphological and Raman spectroscopic characteristics combined with EPMA analyses, iron oxide minerals in sapphire from Southern Vietnam can be grouped into wüstite, hercynite, and ilmenite. These EPMA analyses are newly reported for the mineral chemistry of these oxide inclusions in sapphires from Southern Vietnam.

This is the first report of wüstite found as an inclusion in gem sapphire. Its composition, $\mathrm{Fe}_{1-\mathrm{x}} \mathrm{O}$ with $0.04<\mathrm{x}<0.12$, should be stable at $<570^{\circ} \mathrm{C}$ and can be transformed between $\alpha$-Fe and $\mathrm{Fe}_{3} \mathrm{O}_{4}$ [31]. As reported above, low power laser $(0.5 \mathrm{~mW})$ excitation can decrease alterations on the wüstite surface and then generate a Raman spectrum very 
similar to the pattern of magnetite $\left(\mathrm{Fe}_{3} \mathrm{O}_{4}\right)$ (Figure 6a). This indicates that the structure of wüstite is made up of non-equivalent sites, called non-stoichiometric wüstite $(\mathrm{FeO})$, because the structure of non-equivalent sites of non-stoichiometric wüstite is similar to that of magnetite $\left(\mathrm{Fe}_{3} \mathrm{O}_{4}\right)$ [32]. However, this spectrum changes to a strong decrease in half-intensity peak positions of the magnetite structure $\left(\mathrm{Fe}_{3} \mathrm{O}_{4}\right)$ and clearly displays new peaks of hematite structure $\left(\mathrm{Fe}_{2} \mathrm{O}_{3}\right)$ plus a peak at $595 \mathrm{~cm}^{-1}$ attributed to wüstite, as noticed by Hanesch [23] (Figure 6b) at high laser power ( $5 \mathrm{~mW}$ ). This effect supports that wüstite's surface with a magnetite structure might be transformed into a hematite structure by the activation of high laser power. The irreversible transformation of magnetite to hematite caused by a lowering of the temperature in the natural process is commonly known as matitization [33]:

$$
\mathrm{Fe}_{3} \mathrm{O}_{4} \rightarrow \gamma \mathrm{Fe}_{2} \mathrm{O}_{3} \rightarrow \alpha \mathrm{Fe}_{2} \mathrm{O}_{3}
$$

Besides, a strong increase in the peak around $667 \mathrm{~cm}^{-1}$ (magnetite) at high laser power was visible in the other wüstite inclusions (Figure $6 \mathrm{c}$ ), which may be attributed to the Raman active vibrations of spinel groups [27], indicating more spinel components in the structure. They are comparable to the EPMA analyses that yielded non-stoichiometric wüstite $(\mathrm{FeO})$ and wüstite-hercynite (Table 1). The hercynite component (e.g., 23 percent in sample PT49) obtained in non-stoichiometric wüstites (Table 1) should affect the intensity of such a magnetite peak (Figure 6c).

Raman spectroscopic features of hercynite spinel inclusions clearly belong to the spinel group. Additionally, EPMA analyses yielded the composition of hercynite (Table 2 and Figure 9).

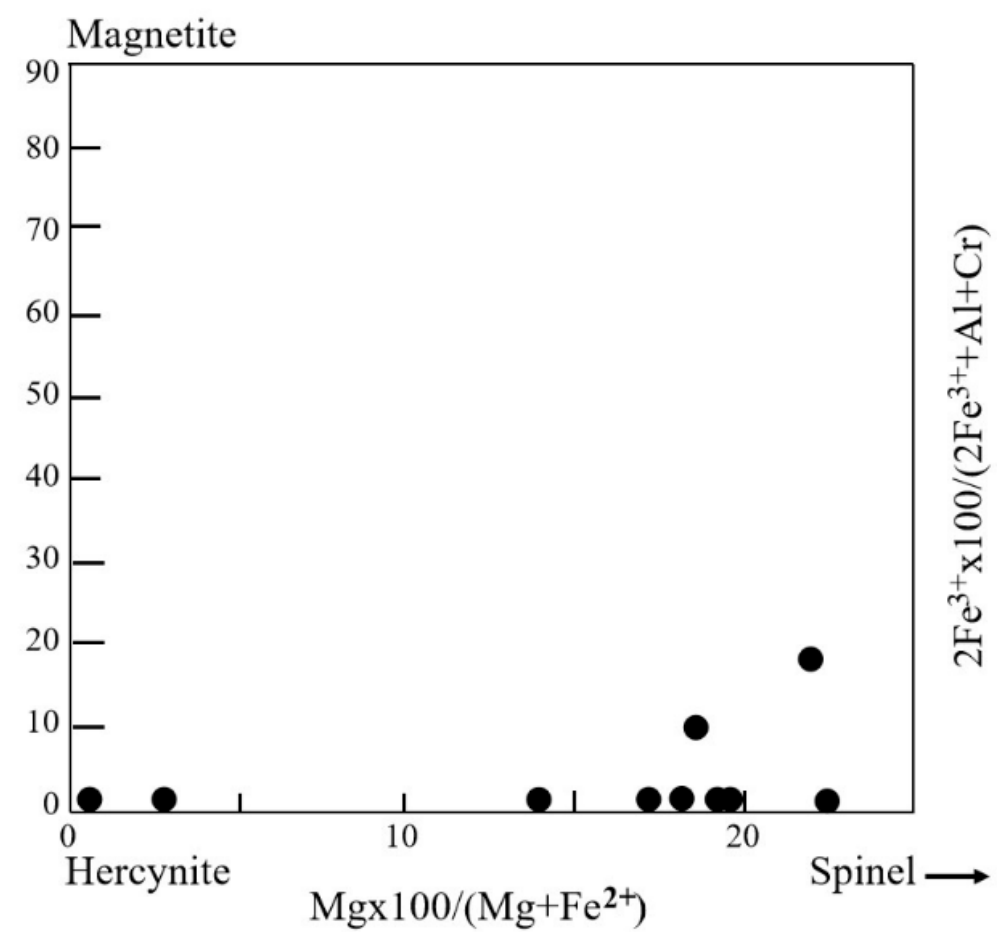

Figure 9. Compositional plots of hercynite spinel inclusions in sapphires from Southern Vietnam. This diagram was suggested by Haggerty [34].

Raman spectroscopic features of ilmenite inclusions only exhibited the mixture patterns of ilmenite and hematite, called ilmenite solid solutions. In addition, the broadened peaks at 399,600 , and $1298 \mathrm{~cm}^{-1}$ of hematite (Figure $8 \mathrm{~b}$ ) were caused by the oxidation of titanomagnetite. This oxidation seems to be influenced by ilmenite exsolution in the Ti-rich magnetite (titanomagnetite) which was reported previously in igneous intrusive from Bijigou, Pazhihua, and Xinjie in China [35]. Meanwhile, the EPMA analyses of ilmenite solid solutions clearly defined titanohematite and titanomagnetite (Table 3 and Figure 10). 


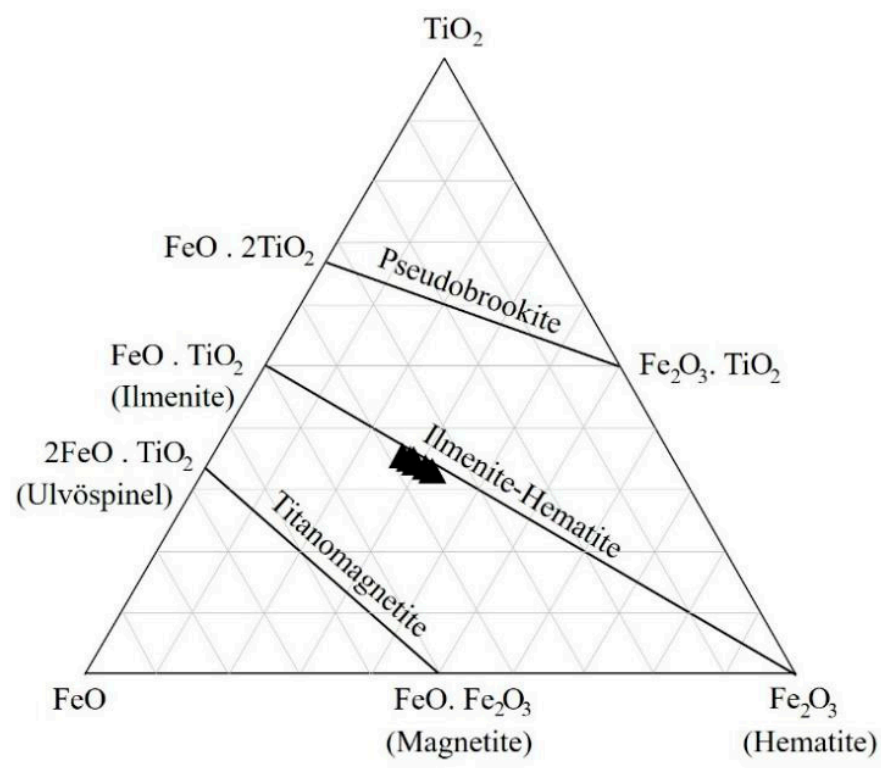

Figure 10. Ternary $\mathrm{FeO}-\mathrm{Fe}_{2} \mathrm{O}_{3}-\mathrm{TiO}_{2}$ diagram and plots of ilmenite-hematite inclusions in sapphire from Southern Vietnam. The diagram was suggested by Buddington and Lindsley [1].

From the results, Raman spectroscopic features of ilmenite inclusions clearly exhibited ilmenite solid solutions (e.g., ilmenohematite and titanomagnetite), which are characteristic of rhombohedral solid solutions [25]; on the other hand, hercynite spinel and wüstite have cubic and octahedral shapes [30]. The EPMA shows attributes of wüstite and hercynite in octahedral iron oxide inclusions, whereas rhombohedral iron oxides favor ilmenitehematite (titanohematite and titanomagnetite). The morphological characteristics of the iron oxide minerals in sapphire from Southern Vietnam match the results of Raman and EPMA analyses.

\subsection{Crystallization Environment}

Iron oxide inclusions in sapphire from Southern Vietnam are mostly characterized by euhedral crystals (rhombohedral and octahedral) which appear to be primary inclusions [36]. Therefore, their chemical and physical conditions can be used to reconstruct the crystallization environment of the host sapphire.

Non-stoichiometric wüstite appears to have formed under a strongly reducing condition $[29,37]$. Furthermore, the co-existing wüstite and hercynite inclusions in sapphire from Southern Vietnam (Figure 5) may be involved by the hercynite's breakdown reaction process [38,39]:

$$
\mathrm{FeAl}_{2} \mathrm{O}_{4} \text { (hercynite) }=\mathrm{Al}_{2} \mathrm{O}_{3} \text { (sapphire } / \text { ruby) }+\mathrm{FeO} \text { (wüstite) }
$$

Therefore, cogenetic wüstite and hercynite inclusions should be formed in a strongly reducing environment related to the hercynite breakdown reaction process. On the other hand, titanomagnetite and titanohematite inclusions should be generated by sub-solidus re-equilibration $[1,40]$ which suggests an oxidizing environment. The compositions of titanomagnetite inclusions vary towards hematite components without ulvöspinel components (Table 3) which should be re-equilibrated under the slow cooling oxidized subsolidus environment $[1,41]$. This supports the complete oxidation reaction of spinel $\mathrm{s}_{\mathrm{ss}}$ by the re-equilibration: $4 \mathrm{Fe}_{3} \mathrm{O}_{4}$ (in exsolution) $+\mathrm{O}_{2}=6 \mathrm{Fe}_{2} \mathrm{O}_{3}$ (in ilmenite) to ilmenite exsolution in titanomagnetite.

This information indicates an environmental change during the formation of these oxide inclusions as well as their host sapphire. Initial crystallization should take place in a strongly reducing (indicated by wüstite occurrences) magma chamber prior to the slow cooling sub-solidus stage under a low oxidizing condition (based on phase transformation 
from titanomagnetite to titanohematite). This process may be related to oxygen fugacity and temperature changing towards the slow cooling process.

Inclusions and their host sapphire from Southern Vietnam were suggested to have formed in the lower crust $[7,9]$ and in the lithospheric mantle [10]. In this study, wüstite with a cubic shape should be typified by crystallization at a temperature of about $570{ }^{\circ} \mathrm{C}$ [31]. This thermal process should be located in the continental crust $\left(\leq 800^{\circ} \mathrm{C}\right)$ [42]. Furthermore, wüstite appears to have occurred in the continental crust, as suggested by Seifert et al. [29], who reported wüstite in fluorapatite crystallized from S-type granite melts. Additionally, hercynite has been recognized in magmatic sapphires also formed in crust [43,44]. In addition, titanomagnetite inclusion with $\mathrm{Il}_{24-30}$ components seems to be formed in plutonic rocks under a crustal environment, as suggested by Buddington et al. [45]. Therefore, coexisting wüstite, hercynite, titanohematite, and titanomagnetite clearly indicate that these oxide inclusions and their host sapphire should have crystallized directly from magma in crust instead of mantle.

Under the silica-saturated condition, iron oxide minerals (i.e., wüstite, hercynite, titanomagnetite, and titanohematite) are not stable; all $\mathrm{Fe}^{2+}$ atoms preferentially enter silicate structures instead. Therefore, wüstite, hercynite, titanomagnetite, and titanohematite may only be formed in the silica-undersaturated environment. Titanomagnetite indicated a higher temperature (at about $600-700^{\circ} \mathrm{C}$ ) than the ilmenite-hematite miscibility gap [46]. Titanomagnetite should crystallize directly from magma prior to sub-solidus reequilibration of iron-titanium oxides during a slow cooling process within a low oxidizing environment. This late state re-equilibration led to a decrease in magnetite in spinel ${ }_{s s}$, with $\mathrm{fO}_{2}$ increasing slightly.

\section{Conclusions}

Iron oxide mineral inclusions provide useful indicators of crystallization conditions of their host sapphire from Southern Vietnam. These iron oxide inclusions include wüstite, hercynite, titanomagnetite, and titanohematite series. Crystal morphology, Raman spectroscopy, and mineral chemical signatures of these iron oxides indicate silicaundersaturated magmatic origin in the lower crust. Wüstite might have crystallized from the hercynite's breakdown reaction (hercynite = sapphire + wüstite), whereas titanomagnetite and titanohematite series should have formed in sub-solidus re-equilibration within the slow cooling process. These results indicate an environmental change during the crystallization process of sapphire, wüstite, and hercynite in the reducing magma chamber prior to a slow cooling sub-solidus re-equilibrium of titanomagnetite to titanohematite with low oxidizing conditions.

Supplementary Materials: The following are available online at https:/ /www.mdpi.com/2075-163 X/11/3/241/s1, Table S1: Sapphire samples used in this study.

Author Contributions: Conceptual and Research Design, D.T.A.V. and C.S.; Methodology, D.T.A.V. and C.S.; Field Work and Sample Collection, D.T.A.V.; Data Analysis, D.T.A.V., C.S., A.S., and A.F.; First Draft Preparation, D.T.A.V.; Reviewing and Editing, D.T.A.V. and C.S. All authors have read and agreed to the published version of the manuscript.

Funding: The first author was supported for her PhD study by "the Scholarship Program for ASIAN Countries Chulalongkorn University".

Institutional Review Board Statement: “Not applicable" for studies not involving humans or animals.

Acknowledgments: We would like to thank the Gem and Jewelry Institute of Thailand (Public Organization) (GIT), Bangkok, Thailand for laboratory and research facilities. Special thanks are expressed to Sopit Poompuang for technical assistance in sample preparation and EPMA analysis at the Geology Department, Faculty of Science, Chulalongkorn University.

Conflicts of Interest: The authors declare no conflict of interest. 


\section{References}

1. Buddington, A.F.; Lindsley, D.H. Iron-Titanium Oxide Minerals and Synthetic Equivalents. J. Petro. 1964, 5, 310-357. [CrossRef]

2. Coenraads, R.; Vichit, P.; Sutherland, F.L. An unusual sapphire-zircon-magnetite xenolith from the Chanthaburi gem province, Thailand. Mineral. Mag. 1995, 59, 465-479. [CrossRef]

3. Karsli, O.; Aydin, F.; Uysal, İ.; Sadiklar, M. Magma Interaction Processes Inferred from Fe-Ti Oxide Compositions in the Dölek and Sar ç̧içek Plutons, Eastern Turkey. Turk. J. Earth Sci. 2008, 17, 297-315.

4. Saminpanya, S.; Sutherland, F.L. Different origins of Thai area sapphire and ruby, derived from mineral inclusions and co-existing minerals. Eur. J. Mineral. 2011, 23, 683-694. [CrossRef]

5. Sutherland, F.L.; Coenraads, R.R. An unusual ruby-sapphire-sapphirine-spinel assemblage from the Tertiary Barrington vocanic province, New South Wales, Australia. Mineral. Mag. 1996, 60, 623-638. [CrossRef]

6. Turnock, A.C.; Eugster, H. Fe Al Oxides: Phase Relationships below $1000{ }^{\circ}$ C. J. Petro. 1962, 3, 533-565. [CrossRef]

7. Izokh, A.E.; Smirnov, S.Z.; Egorova, V.V.; Anh, T.T.; Kovyazin, S.V.; Phuong, N.T.; Kalinina, V.V. The conditions of formation of sapphire and zircon in the areas of alkali-basaltoid volcanism in Central Vietnam. Russ. Geol. Geophys. 2010, 51, 719-733. [CrossRef]

8. Smith, C.P.; Kammerling, R.C.; Keller, A.S.; Peretti, A.; Scarratt, K.V.; Khoa, N.D.; Repetto, S. Sapphires from Southern Vietnam. G. Gemol. 1995, 31, 168-186. [CrossRef]

9. Vu, D.T.A.; Salam, A.; Funka, A.; Blousova, E.; Sutthirat, C. Mineral inclusions in sapphire from basaltic terranes in southern Vietnam: Indicator of formation model. G. Gemol. 2020, 56. (in press).

10. Vuong, B.T.S.; Osanai, Y.; Lenz, C.; Nakano, N.; Adachi, T.; Belousova, E.; Kitano, I. Gem-Quality Zircon Megacrysts from Placer Deposits in the Central Highlands, Vietnam-Potential Source and Links to Cenozoic Alkali Basalts. Minerals $2019,9,89$.

11. Tri, T.V.; Khuc, V. Geology and Earth Resources of Vietnam; General Department of Geology and Minerals of Vietnam: Ha Noi, Vietnam; Publishing House for Science and Technology: Ha Noi, Vietnam, 2011; p. 634.

12. Hoa, T.T.; Phuong, N.T.; Anh, T.T.; Van, V.V.; Hoang, N.; Thanh, H.H.; Anh, P.L.; Nien, B.A.; Hung, T.Q.; Dung, P.T.; et al. Study of forming conditions and distribution laws of precious and rare minerals related to magmatic activity in Central Vietnam and Tay Nguyen Highlands. In State-Level Project, Code DTDL-2003/07; Publishing House for Science and Technology: Ha Noi, Vietnam, 2005; Volume 1, p. 347. (in Vietnamese)

13. Hutchison, C. Tectonic evolution of Southeast Asia. Bull. Geol. Soc. Malaysia 2014, 60, 1-18. [CrossRef]

14. Hutchison, C.S. Geological Evolution of South-East Asia; Clarendon Press: Oxford, UK, 1989.

15. Metcalfe, I. Palaeozoic-Mesozoic history of SE Asia. Geol. Soc. Lond. Spec. Publ. 2011, 355, 7-35. [CrossRef]

16. Barr, S.; MacDonald, A. Geochemistry and geochronology of late Cenozoic basalts of Southeast Asia: Summary. Geol. Soc. Am. Bull. 1981, 92, 1069-1142. [CrossRef]

17. Hoang, N.; Flower, M.; Chi, C.; Xuan, P.; Quy, H.; Son, T. Collision-induced basalt eruptions at Pleiku and Buôn Mê Thuôt, south-central Viet Nam. J. Geodyn. 2013, 69, 65-83. [CrossRef]

18. Hoang, N.; Flower, M.; Nguyen, X.; Nguyen, T. Trace element and isotopic compositions of Vietnamese basalts: Implications for mantle dynamics in the southeast Asian region. Bull. Soc. Géol. France 1996, 167, 785-795.

19. Anh, H.T.H.; Choi, S.H.; Yu, Y.; Hieu, P.T.; Hoang, N.K.; Ryu, J. Geochemical constraints on the spatial distribution of recycled oceanic crust in the mantle source of late Cenozoic basalts, Vietnam. Lithos 2018, 296, 382-395.

20. Hoang, N.; Flower, M. Petrogenesis of Cenozoic Basalts from Vietnam: Implication for Origins of a 'Diffuse Igneous Province'. J. Petro. 1998, 39, 369-395. [CrossRef]

21. Garnier, V.; Ohnenstetter, D.; Giuliani, G.; Fallick, A.E.; Trinh, P.; Quang, V.; Van, L.; Schwarz, D. Basalt petrology, zircon ages and sapphire genesis from Dak Nong, southern Vietnam. Mineral. Mag. 2005, 69, 21-38. [CrossRef]

22. Faria, D.L.A.; Silva, S.V.; Oliveira, M.T.M. Raman microspectroscopy of some iron oxides and oxyhydroxides. J. Raman Spectrosc. 1997, 28, 873-878. [CrossRef]

23. Hanesch, M. Raman spectroscopy of iron oxides and (oxy)hydroxides at low laser power and possible applications in environmental magnetic studies. Geophys. J. Int. 2009, 177, 941-948. [CrossRef]

24. Shebanova, O.; Lazor, P. Raman Study of Magnetite $\left(\mathrm{Fe}_{3} \mathrm{O}_{4}\right)$ : Laser-Induced Thermal Effects and Oxidation. J. Raman Spectrosc. 2003, 34, 845-852. [CrossRef]

25. Wang, A.; Kuebler, K.; Jolliff, B.; Haskin, L. Raman spectroscopy of Fe-Ti-Cr-Oxides, case study: Martian meteorite EETA79001. Am. Mineral. 2004, 89, 665-680. [CrossRef]

26. Droop, G.T.R. A general equation for estimating Fe3+ concentrations in ferromagnesian silicates and oxides from microprobe analyses, using stoichiometric criteria. Mineral. Mag. 1987, 51, 431-435. [CrossRef]

27. Cynn, H.; Sharma, S.K.; Cooney, T.F.; Nicol, M. High-temperature Raman investigation of order-disorder behavior in the $\mathrm{MgAl}_{2} \mathrm{O}_{4}$ spinel. Phys. Rev. B Condens. Matter. 1992, 45, 500-502. [CrossRef]

28. Ospitali, F.; Sabetta, T.; Tullini, F.; Nannetti, M.; Lonardo, G. The role of Raman microspectroscopy in the study of black gloss coatings on Roman pottery. J. Raman Spectrosc. 2005, 36, 18-23. [CrossRef]

29. Seifert, W.; Thomas, R.; Rhede, D.; Förster, H.J. Origin of coexisting wüstite, Mg-Fe and REE phosphate minerals in graphitebearing fluorapatite from the Rumburk granite. Eur. J. Mineral. 2010, 22, 495-507. [CrossRef]

30. Smyth, J.; Jacobsen, S.; Hazen, R. Comparative crystal chemistry of dense oxide minerals. Rev. Mineral. Geochem. 2000, 41, 157-186. [CrossRef] 
31. Jozwiak, W.; Kaczmarek, E.; Maniecki, T.; Ignaczak, W.; Maniukiewicz, W. Reduction behavior of iron oxides in hydrogen and carbon monoxide atmospheres. Appl. Catal. A-Gen. 2007, 326, 17-27. [CrossRef]

32. Koch, F.B.; Fine, M.E. Magnetic Properties of FexO as Related to the Defect Structure. J. Appl. Physics 1967, 38, 1470-1471. [CrossRef]

33. Romdohr, P. The Ore Minerals and Their Intergrowths; Pergamon Press: Toronton, ON, Canada, 1969.

34. Haggerty, S.E. Oxide mineralogy of the upper mantle. In Oxide Minerals: Petrologic and Magnetic Significance; Lindley, D.H., Ed.; Mineralogical Society of America: Washington, DC, USA, 1991; pp. 355-416.

35. Tan, W.; Liu, P.; He, H.; Wang, C.; Liang, X. Mineralogy and Origin of Exsolution In Ti-Rich Magnetite from Different Magmatic Fe-Ti Oxide-Bearing Intrusions. Can. Mineral. 2016, 54, 539-553. [CrossRef]

36. Gübelin, E.J. Internal World of Gemstones; ABC Edition: Zurich, Switzerland, 1983; p. 234.

37. Miyashiro, A. Oxidation and reduction in the Earth's crust with special reference to the role of graphite. Geochim. Cosmochim. Acta 1964, 28, 717-729. [CrossRef]

38. Nestola, F.; Periotto, B.; Anzolini, C.; Andreozzi, G.; Woodland, A.; Lenaz, D.; Alvaro, M.; Princivalle, F. Equation of state of hercynite, $\mathrm{FeAl}_{2} \mathrm{O}_{4}$, and high-pressure systematics of $\mathrm{Mg}-\mathrm{Fe}-\mathrm{Cr}-\mathrm{Al}$ spinels. Mineral. Mag. 2015, 79, 285-294. [CrossRef]

39. Schollenbruch, K.; Woodland, A.B.; Frost, D.J. The stability of hercynite at high pressures and temperatures. Phys. Chem. Minerals 2010, 37, 137-143. [CrossRef]

40. Lattard, D. Experimental evidence for the exsolution of ilmenite from titaniferous spinel. Am. Mineral. 1995, 80, 968-981. [CrossRef]

41. Vincent, E.A. Ulvöspinel in the Skaergaard intrusion, Greenland. Neues Jahrb. Mineral. Abh. 1960, 94, $993-1016$.

42. Jeanloz, R.; Morris, S. Temperature Distribution in the Crust and Mantle. Annu. Rev. Earth Planet. Sci. 1986, 14, $377-415$. [CrossRef]

43. Sutherland, F.L.; Hoskin, P.; Fanning, C.; Coenraads, R. Models of corundum origin from alkali basaltic terrains: A reappraisal. Contrib. Miner. Petrol. 1998, 133, 356-372. [CrossRef]

44. Sutherland, F.L.; Bosshart, G.; Fanning, C.; Hoskin, P.W.O.; Coenraads, R. Sapphire crystallization, age and origin, Ban Huai Sai, Laos: Age based on zircon inclusions. J. Asian Earth Sci. 2002, 20, 841-849. [CrossRef]

45. Buddington, A.F.; Fahey, J.J.; Vlisidis, A.C. Thermometric and petrogenetic significance of titaniferous magnetite. Am. J. Sci. 1955, 253, 497-532. [CrossRef]

46. Lindsley, D.H. Experimental studies of oxide minerals. Rev. Mineral. Geochem. 1991, 25, 69-106. 